\title{
Wide-Band Spatially Distributed TE10 Substrate Integrated Waveguide Transition for High-Power Generation at mm-Wave Frequencies
}

\author{
Artem Roev ${ }^{\# 1}$, Rob Maaskant ${ }^{\#, \uparrow 2}$, Marianna Ivashina ${ }^{\# 3}$, and Anders Höök ${ }^{* 4}$ \\ \# Department of Signals and Systems, Chalmers University of Technology, Gothenburg, Sweden \\ + Electromagnetics Group, Eindhoven University of Technology, The Netherlands \\ ${ }^{1}$ roev@chalmers.se \\ 22rob.maaskant@chalmers.se |r.maaskant@tue.nl \\ ${ }^{3}$ marianna.ivashina@chalmers.se \\ * SAAB AB, Gothenburg, Sweden \\ 4 anders.hook@saabgroup.com
}

\begin{abstract}
An array of microstrip lines is used for the direct excitation of the spatially distributed TE10 substrate integrated waveguide (SIW) mode. The proposed configuration can generate $4 \mathrm{x}$ more power with a $4 \mathrm{x}$ smaller form factor relative to a single microstrip-to-SIW transition, while offering a larger bandwidth. This is an important step toward the generation of high power in densely integrated mm-wave antenna systems.
\end{abstract}

Keywords-Substrate integrated waveguide; spatial power combining; mm-wave systems.

\section{INTRODUCTION}

The generation and transmission of high power is a major challenge at mm-wave frequencies, since the atmospheric propagation and material losses are significant and increase as frequency increases. This is further exacerbated by the fact that semiconductors reduce in size and deliver less power when moving up into the mm-wave frequency region. Hence, more transistors are needed to overcome these limitations, but will collectively generate more heat. These problems must be overcome to, for instance, enable the high data throughputs that are envisioned for future $5 \mathrm{G}$ communication systems.

Substrate integrated waveguide (SIW) technology offers a low-cost low-profile mm-wave integration solution. However, a transition is needed to interface the principal SIW electromagnetic field mode to a single network node, or TEMtype transmission line (TL), so that a circuit component can be connected. A typically employed microstrip-to-SIW transition is shown in Fig. 1(a), where a tapered section is proposed to transform the distributed SIW to the spatially confined microstrip TL mode [1], [2].

We propose a transition that is more compact (no TL tapering needed) by interfacing an array of amplifiers to a single SIW port. This allows to directly feed the spatially distributed TE10 single SIW mode with high power. Note that this is different from the multi-mode concept in [3]. Our transition also exhibits larger impedance bandwidth characteristics. The proposed concept is illustrated in Fig. 1(b).

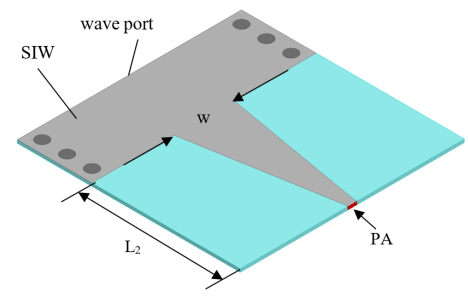

(a)

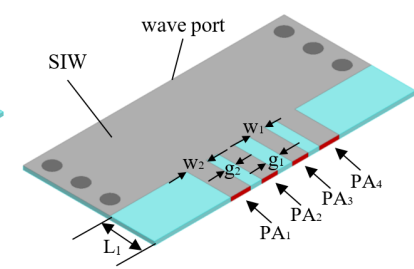

(b)
Fig. 1: (a) Classical single microstrip-to-SIW transition; (b) Proposed multi-channel transition and optimization parameters.

\section{Design DETAILS}

Our design aims at operating in the $\mathrm{K} / \mathrm{Ka}$-Band and is based on the proposed concept in Fig. 1(b) with indicated geometrical parameters. The structure is built on an RT5880 laminate with substrate thickness $0.127 \mathrm{~mm}$ and relative dielectric constant $\varepsilon_{r}=2.2$. The low dissipation factor $(\tan \delta @ 10 \mathrm{GHz}=0.0009)$, thereby extending the applicability of RT5880 to Ka-band and above. Our specific design employs four parallel microstrip lines, each of which is terminated by $50 \mathrm{Ohm}$ to represent the output resistance of a power amplifier. To expedient the simulations, the SIW vias were replaced by conductive side walls. Perfect Electric Conductors were used for the metals. Regarding Fig. 1, the optimized designs for best impedance match are (in mm): $w=2.4 ; L_{2}=6.5 ; L_{1}=1.54 ; w_{1}=0.54 ; w_{2}=0.68$; $g_{1}=0.5 ; g_{2}=0.39$. The SIWs are identical, width=7.11.

\section{NUMERICAL RESULTS}

Fig. 2 shows the field propagation inside the SIW and the coupled fields to the microstrip TLs when the SIW port is excited by the TE10 mode (i.e. receive mode). When transmitting, the active reflection coefficients of the microstrip TLs should be considered due to the strong electromagnetic coupling between them. The simulated active and passive 


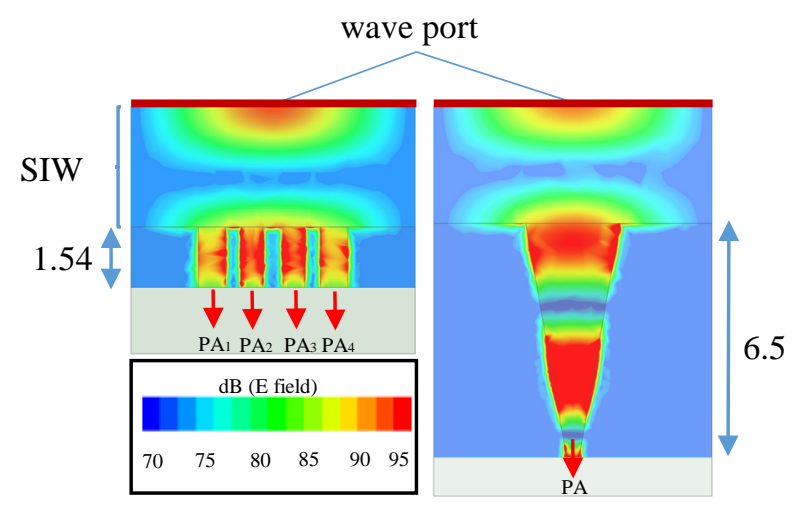

Fig. 2. Magnitude of $E$-field distribution at $31.5 \mathrm{GHz}$ (real part) and sizes $(\mathrm{mm})$ of the proposed multi-channel transition and the conventional single-channel tapered microstrip transition.

wave port reflection coefficients of both the proposed transition and the conventional single-channel tapered microstrip TL are shown in Fig. 3. The simulated multichannel transition demonstrates a wide bandwidth performance relative to its single-channel counterpart.

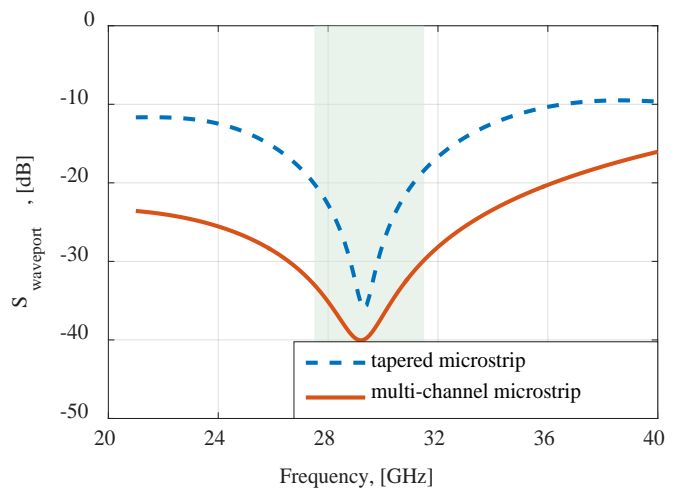

Fig. 3. Simulated wave port reflection coefficient of the proposed multi-channel transition and conventional single-channel tapered microstrip transition.

The simulated active $\left|\Gamma_{i}\right|$ and passive $\left|S_{i i}\right|$ reflection coefficient of the $50-\Omega$ microstrip ports are shown in Fig. 4. The active reflection coefficients are better than $-30 \mathrm{~dB}$ over the desired frequency range $(27.5-31.5 \mathrm{GHz})$.

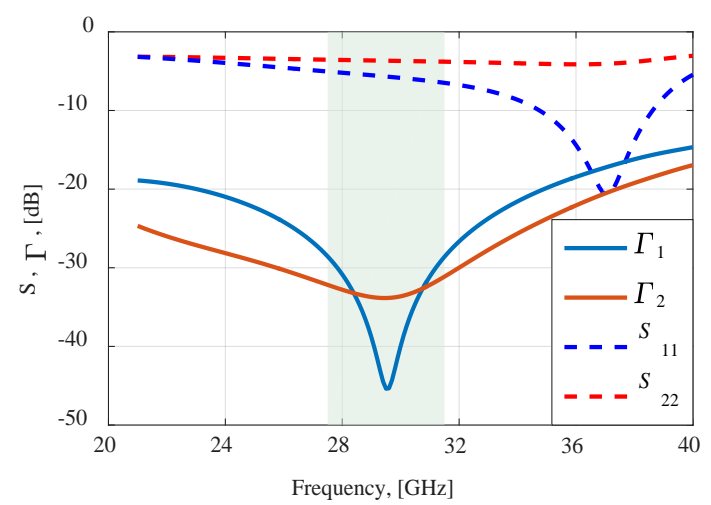

Fig. 4. Simulated active and passive (dashed lines) reflection coefficient of the $50 \Omega$ microstrip ports of the proposed transition.
The designed four-channel transition is almost four times more compact (Fig. 2) and allows to generate four times higher mmwave output power as compared to a conventional tapered microstrip transition.

Fig. 5 shows the microstrip port powers when the wave port is excited by $1 \mathrm{~W}$ (i.e. receive mode). The power distribution is near uniform (i.e. < 25\% variation, $250 \mathrm{~mW}$ is ideal), implying that the power amplifiers in the array are utilized effectively.

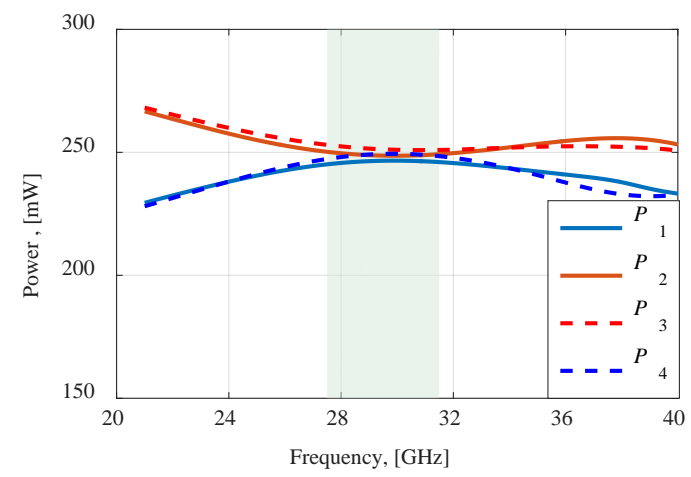

Fig. 5. Simulated power accepted by the microstrip ports when the wave port is excited by $1 \mathrm{~W}$.

\section{CONCLUSIONS}

A novel transition concept based on the direct excitation of the TE10 substrate integrated waveguide mode by an array of strongly coupled microstrip lines has been proposed. The simulated active reflection coefficients of the four-channel transition at their microstrip ports and passive reflection coefficient at the wave port of a single transition are better than $-30 \mathrm{~dB}$ over the desired frequency range $(27.5-31.5 \mathrm{GHz})$. The quad-channel transition is four times more compact and allows to generate four times higher mm-wave output power compared to conventional tapered microstrip transitions. Its relatively short length allows to decreases transition losses and renders the structure suitable for much higher frequencies. The concept is expected to play a determining role in future mmwave communication systems where both high-power generation and low-loss structures are required.

\section{ACKNOWLEDGMENT}

The work is a part of the «Silicon-based Ka-band massive MIMO antenna systems for new telecommunication services (SILIKA)» project, funded by the European Union's Horizon 2020 research and innovation program under the Marie Skłodowska Curie grant agreement \#721732.

\section{REFERENCES}

[1] D. Deslandes, "Design equations for tapered microstrip-to-substrate integrated waveguide transitions“. IEEE MTT-S Int. Microwave Symp. Digest, Anaheim, CA, USA, 23-28 May 2010, pp. 704-707.

[2] Z.X. Wang, P.G. Su, L. Shang and W.B. Dou, "A Waveguide-toMicrostrip Transition at W-band”, International Conference on Microwave and Millimeter Wave Technology (ICMMT), May 2010, pp. 1486-1489.

[3] A. Suntives and R. Abhari, "Design and application of multimode substrate integrated waveguides in parallel multichannel signaling systems,” IEEE Trans. Microw. Theory Techn., vol. 57, no. 2009. pp 1563-1571. 